\title{
AUTOMATED ACHROMATIC CONTRAST AND CHROMATIC DISCRIMINATION SENSITIVITY TESTING IN DYSTHYROID OPTIC NEUROPATHY
}

\author{
V. TANNER ${ }^{1}$, S. J. TREGEAR ${ }^{1,2}$, L. G. RIPLEY ${ }^{2}$ and S. F. VICKERS ${ }^{1}$ \\ Brighton
}

\begin{abstract}
SUMMARY
Our experience of patients with dysthyroid eye disease shows that normal chromatic discrimination sensitivity precludes the diagnosis of optic nerve compression (31 patients), and that clinically confirmed optic nerve compression is invariably associated with decreased chromatic discrimination sensitivity thresholds (8 patients). Dysthyroid patients enrolled in this study underwent automated achromatic contrast and chromatic discrimination sensitivity testing on presentation, with repeat assessment of those patients suspected of developing optic nerve compression. If chromatic discrimination sensitivity was decreased, patients were followed up more frequently. If abnormal chromatic discrimination sensitivity was accompanied by a relative afferent pupillary defect (RAPD) or decreased Snellen visual activity (VA), then optic nerve decompression was performed. The automated chromatic discrimination sensitivity test described represents a quick, reproducible and cheap clinical test which we feel is of value in assessing patients with dysthroid eye disease. We suggest that sequential chromatic discrimination sensitivity assessment is a sensitive and effective way of monitoring patients at risk of dysthyroid optic neuropathy.
\end{abstract}

Thyroid-related eye disease is a distressing condition occurring in association with Graves' hyperthyroidism in $90 \%$ of cases. ${ }^{1}$ The two main causes of visual loss associated with thyroid eye disease are dysthyroid optic neuropathy (DON) and corneal ulceration. The former of these two conditions poses the greater diagnostic dilemma to the ophthalmologist since it is often insidious in nature and minimally symptomatic in its early stages. Of those patients suffering from dysthyroid eye disease, approximately $5-10 \%$ will

From: ${ }^{1}$ Sussex Eye Hospital, Brighton, East Sussex. ${ }^{2}$ Graduate Division of Biomedical Engineering, University of Sussex, Falmer, Brighton, East Sussex, UK.

Correspondence to: Miss S. F. Vickers, Sussex Eye Hospital, Eastern Road, Brighton, East Sussex BN2 5BF, UK. develop associated optic nerve compression. ${ }^{2}$

Traditional methods used to assess compression of the optic nerve include Snellen visual acuity, the presence of a relative afferent pupillary defect (RAPD), screening for red/green colour vision deficits using the Ishihara plates, and examination of the optic nerve head. Unfortunately, none of the above indicators is invariably present in association with optic nerve compression. An extensive review of dysthyroid patients by Trobe $e t a l^{2}$ demonstrated that up to $50 \%$ of those with DON have unremarkable optic discs, and that Ishihara test scores are significantly decreased only if visual acuities are below 20/40. In this study we have assessed the viability of automated achromatic contrast sensitivity and chromatic discrimination sensitivity as possible tests for the early diagnosis of optic nerve compression. It is known that achromatic contrast sensitivity is diminished in optic nerve compression ${ }^{3-5}$ and a report exists of decreased colour sensitivity associated with DON. ${ }^{6}$

\section{PATIENTS AND METHODS}

Thirty-nine patients were entered into the study with varying grades of dysthyroid eye disease. Mean age was 57 years (range 30-79 years) and mean duration of disease was 85 months (range $0-480$ months). All patients presented to the Sussex Eye Hospital over a 3-year period (January 1991 to January 1994) and were documented as biochemically hyperthyroid.

All patients were reviewed regularly by one experienced ophthalmologist using a standard clinical format. A full ophthalmic examination was carried out which included Snellen visual acuity, corneal and conjunctival appearance, presence of a RAPD, degree of lid retraction, exophthalmometer reading, orthoptic assessment, Ishihara colour plate performance and optic disc appearance. Orbital CT scanning was performed in those patients with suspected optic nerve compression. All patients 
Table I. NOSPECS classification of thyroid eye disease

\begin{tabular}{ll}
\hline Grade & Description \\
\hline 0 & No ocular symptoms \\
1 & Only signs, no symptoms. Signs limited to upper lid retraction with or without lid lag and mild proptosis \\
2 & Soft tissue involvement: oedema of conjunctivae or lids, extrusion of orbital fat \\
3 & Proptosis: mild, $21-23 \mathrm{~mm}$; moderate, $24-27 \mathrm{~mm}$; marked, $>28 \mathrm{~mm}$ \\
4 & Extrinsic muscle involvement \\
5 & Corneal involvement: minimal, corneal stippling; moderate, ulceration; marked, necrosis and perforation \\
6 & Sight loss secondary to optic nerve involvement \\
\hline
\end{tabular}

underwent automated achromatic contrast sensitivity and chromatic discrimination sensitivity testing. These tests were repeated at increased intervals if optic nerve compression was suspected clinically, or if the initial achromatic contrast and chromatic discrimination test results were abnormal.

The severity of thyroid eye disease present in each patient was graded using the NOSPECS classification as originally described by Werner et $a l^{7}$ and summarised in Table I.

Optic nerve compression was diagnosed on the basis of a decrease in best corrected Snellen visual acuity of one line or more plus one of the following: definite RAPD, optic disc oedema or poor performance on the Ishihara plates.

Both achromatic contrast sensitivity and chromatic discrimination sensitivity were measured using a cathode ray tube system under software control which has been described elsewhere. ${ }^{8-11}$ The tests were performed under ambient lighting conditions with the patient seated $2 \mathrm{~m}$ from the screen: the stimuli at this distance subtend the central $4^{\circ}$ of vision. Achromatic contrast sensitivity was measured at six spatial frequencies $(0.33,0.66,2.20,3.40,10.0$

Table II. Summary of data obtained from the 39 thyroid eye disease patients

\begin{tabular}{|c|c|c|c|c|c|c|c|c|c|c|c|c|}
\hline $\begin{array}{c}\text { Subject } \\
\text { no. }\end{array}$ & Age (yr) & Eye & $\begin{array}{l}\text { Snellen } \\
\text { VA }\end{array}$ & $\begin{array}{c}\text { NOSPECS } \\
\text { grade }\end{array}$ & $0.33 \mathrm{cpd}$ & $0.66 \mathrm{cpd}$ & $2.20 \mathrm{cpd}$ & $3.40 \mathrm{cpd}$ & $10.0 \mathrm{cpd}$ & $17.0 \mathrm{cpd}$ & RGDS & TDS \\
\hline 1 & 30 & $R$ & $6 / 9$ & 6 & 0.52 & 1.11 & 0.94 & 1.01 & 0.32 & 0.17 & 0.43 & 0.33 \\
\hline 2 & 71 & $\mathrm{R}$ & $6 / 12$ & 6 & 0.93 & 1.48 & 1.94 & 1.67 & 0.98 & 0.59 & 0.28 & 0.49 \\
\hline 3 & 53 & L & $6 / 6$ & 6 & 1.05 & 1.81 & 2.28 & 2.13 & 1.69 & 1.39 & 0.50 & 0.47 \\
\hline 4 & 62 & L & $6 / 9$ & 6 & 1.04 & 1.25 & 1.72 & 1.64 & 0.52 & 0.21 & 0.39 & 0.22 \\
\hline 5 & 62 & $\mathrm{R}$ & $6 / 9$ & 6 & 1.04 & 1.24 & 1.84 & 1.87 & 1.05 & 0.40 & 0.63 & 0.43 \\
\hline 6 & 58 & $\mathrm{R}$ & $6 / 9$ & 5 & 1.41 & 1.69 & 2.25 & 2.28 & 1.71 & 1.05 & 0.82 & 0.79 \\
\hline 7 & 48 & $\mathrm{R}$ & $6 / 5$ & 5 & 1.08 & 1.47 & 2.26 & 2.28 & 1.64 & 0.75 & 0.65 & 0.44 \\
\hline 8 & 79 & $\mathrm{R}$ & $6 / 5$ & 5 & 1.16 & 1.82 & 2.00 & 2.05 & 1.40 & 0.53 & 0.77 & 0.83 \\
\hline 9 & 69 & L & $6 / 12$ & 5 & 1.38 & 1.72 & 2.02 & 1.98 & 1.24 & 0.62 & 0.75 & 0.76 \\
\hline 10 & 59 & $\mathrm{R}$ & $6 / 5$ & 5 & 1.08 & 1.52 & 1.90 & 1.87 & 1.52 & 1.02 & 0.81 & 0.79 \\
\hline 11 & 68 & $\mathrm{R}$ & $6 / 6$ & 5 & 1.38 & 1.88 & 2.44 & 2.25 & 1.27 & 0.43 & 0.55 & 0.45 \\
\hline 12 & 53 & L & $6 / 5$ & 5 & 1.57 & 1.88 & 1.95 & 1.90 & 1.30 & 1.00 & 0.80 & 0.81 \\
\hline 13 & 46 & $\bar{L}$ & $6 / 9$ & 5 & 1.19 & 1.53 & 2.12 & 2.16 & 1.49 & 0.67 & 0.74 & 0.63 \\
\hline 14 & 67 & $\mathrm{R}$ & $6 / 6$ & 5 & 1.29 & 1.78 & 2.41 & 2.37 & 2.03 & 1.22 & 0.74 & 0.71 \\
\hline 15 & 63 & L & $6 / 5$ & 5 & 1.25 & 1.53 & 2.14 & 2.04 & 1.53 & 0.78 & 0.49 & 0.34 \\
\hline 16 & 38 & $\mathrm{~L}$ & $6 / 6$ & 4 & 1.54 & 1.70 & 2.32 & 2.35 & 2.00 & 1.43 & 0.73 & 0.68 \\
\hline 17 & 37 & $\mathrm{R}$ & $6 / 6$ & 4 & 1.51 & 1.91 & 2.35 & 2.47 & 1.84 & 0.92 & 0.76 & 0.78 \\
\hline 18 & 63 & L & $6 / 9$ & 4 & 1.13 & 1.62 & 2.22 & 2.18 & 1.13 & 0.76 & 0.65 & 0.52 \\
\hline 19 & 56 & $\overline{\mathrm{L}}$ & $6 / 9$ & 4 & 1.24 & 1.56 & 2.03 & 1.96 & 1.02 & 0.21 & 0.62 & 0.61 \\
\hline 20 & 57 & L & $6 / 6$ & 4 & 1.52 & 1.91 & 2.73 & 2.68 & 2.30 & 1.82 & 0.89 & 0.74 \\
\hline 21 & 58 & $\mathrm{R}$ & $6 / 6$ & 4 & 1.30 & 1.54 & 2.22 & 2.42 & 1.84 & 1.22 & 0.83 & 0.93 \\
\hline 22 & 77 & L & $6 / 9$ & 4 & 1.10 & 1.41 & 2.09 & 2.03 & 0.81 & 0.72 & 0.69 & 0.74 \\
\hline 23 & 76 & L & $6 / 6$ & 4 & 1.19 & 1.48 & 2.09 & 2.25 & 1.47 & 0.75 & 0.66 & 0.60 \\
\hline 24 & 73 & $\bar{L}$ & $6 / 9$ & 4 & 1.12 & 1.54 & 1.94 & 1.89 & 1.47 & 0.92 & 0.68 & 0.63 \\
\hline 25 & 57 & $\mathrm{R}$ & $6 / 6$ & 4 & 1.48 & 1.89 & 2.42 & 2.42 & 1.84 & 1.32 & 0.83 & 0.81 \\
\hline 26 & 69 & $\mathrm{R}$ & $6 / 6$ & 4 & 1.27 & 1.62 & 2.27 & 2.16 & 1.09 & 0.84 & 0.66 & 0.67 \\
\hline 27 & 43 & L & $6 / 6$ & 4 & 1.35 & 1.64 & 2.28 & 2.25 & 1.57 & 0.92 & 0.56 & 0.69 \\
\hline 28 & 59 & $\mathrm{R}$ & $6 / 4$ & 4 & 1.26 & 1.59 & 2.32 & 2.47 & 1.98 & 1.51 & 0.66 & 0.63 \\
\hline 29 & 66 & L & $6 / 4$ & 4 & 1.29 & 1.71 & 2.12 & 2.09 & 1.39 & 1.13 & 0.69 & 0.72 \\
\hline 30 & 50 & $\bar{L}$ & $6 / 5$ & 4 & 1.76 & 1.94 & 2.52 & 2.50 & 2.00 & 1.43 & 0.69 & 0.76 \\
\hline 31 & 48 & $\mathrm{R}$ & $6 / 5$ & 4 & 1.79 & 1.96 & 2.48 & 2.54 & 2.12 & 1.44 & 0.80 & 0.79 \\
\hline 32 & 61 & $\mathrm{R}$ & $6 / 6$ & 3 & 1.26 & 1.69 & 2.09 & 2.28 & 1.52 & 1.00 & 0.68 & 0.54 \\
\hline 33 & 45 & L & $6 / 5$ & 3 & 1.46 & 1.63 & 2.30 & 2.25 & 1.73 & 1.35 & 0.66 & 0.71 \\
\hline 34 & 36 & $\bar{L}$ & $6 / 5$ & 3 & 1.45 & 1.91 & 2.47 & 2.53 & 2.16 & 1.57 & 0.79 & 0.86 \\
\hline 35 & 57 & $\mathrm{R}$ & $6 / 9$ & 3 & 1.71 & 1.91 & 2.22 & 2.13 & 1.24 & 0.92 & 0.70 & 0.56 \\
\hline 36 & 51 & L & $6 / 5$ & 2 & 1.55 & 1.96 & 2.50 & 2.57 & 2.28 & 1.73 & 0.80 & 0.73 \\
\hline 37 & 44 & R & $6 / 5$ & 1 & 1.51 & 1.85 & 2.47 & 2.44 & 2.16 & 1.57 & 0.79 & 0.84 \\
\hline 38 & 41 & $\mathrm{~L}$ & $6 / 6$ & 2 & 1.59 & 1.89 & 2.32 & 2.37 & 1.64 & 1.21 & 0.74 & 0.71 \\
\hline 39 & 45 & $\mathrm{~L}$ & $6 / 6$ & 2 & 1.55 & 1.92 & 2.32 & 2.42 & 1.92 & 1.52 & 0.79 & 0.84 \\
\hline
\end{tabular}

cpd, cycles per degree; RGDS, red-green discrimination sensitivity; TDS, Tritan Discrimination Sensitivity. 
Table III. Statistical significance of achromatic contrast sensitivity and chromatic discrimination sensitivity scores for each thyroid grade group versus normal, age-matched controls

\begin{tabular}{lcccccccc}
\hline NOSPECS grade & $0.33 \mathrm{cpd}$ & $0.66 \mathrm{cpd}$ & $2.20 \mathrm{cpd}$ & $3.40 \mathrm{cpd}$ & $10.0 \mathrm{cpd}$ & $17.0 \mathrm{cpd}$ & RGDS & TDS \\
\hline 1,2 and 3 & -3.69 & -5.91 & -16.94 & -7.35 & 0.57 & 4.74 & 13.38 & 17.88 \\
& $\mathrm{NS}$ & $\mathrm{NS}$ & $\mathrm{NS}$ & $\mathrm{NS}$ & $\mathrm{NS}$ & $\mathrm{NS}$ & NS & NS \\
4 & -36.41 & -39.73 & -33.73 & -24.07 & -25.72 & -25.65 & -19.73 & -8.7 \\
& $p=0.0302$ & $p=0.0107$ & $\mathrm{NS}$ & $\mathrm{NS}$ & $\mathrm{NS}$ & $\mathrm{NS}$ & $\mathrm{NS}$ & $\mathrm{NS}$ \\
5 & -49.89 & -43.17 & -48.8 & -52.56 & -40.86 & -53.03 & -2.55 & -6.37 \\
& $p=0.0037$ & $p=0.0243$ & $p=0.0051$ & $p=0.0016$ & $p=0.0439$ & $p=0.0014$ & NS & NS \\
6 & -76.49 & -65.57 & -70.60 & -73.21 & -65.56 & -56.28 & -117.6 & -117.52 \\
& $p=0.0007$ & $p=0.0074$ & $p=0.0026$ & $p=0.0015$ & $p=0.0076$ & $p=0.0456$ & $p=0.0047$ & $p=0.0047$ \\
\hline
\end{tabular}

Significance levels were found using the Kruskal-Wallis non-parametric analysis of variance test. Significance level was set at $p<0.05$. NS, not significant; cpd, cycles per degree; RGDS, Red Green Discrimination Sensitivity; TDS, Tritan Discrimination Sensitivity.

and 17.0 cycles per degree (cpd)). Chromatic discrimination sensitivities were obtained for departures from neutral white (CIE coordinates: 0.33, 0.33 ) along red/green (constant S-cone) and tritan (constant M/L-cone) confusion axes. Thresholds were measured using a randomised double-staircase-reversal algorithm. ${ }^{12}$ The complete test took an average of approximately 10 minutes per eye.

Criteria for exclusion from the study included evidence of any other ocular pathology, a history of systemic disease known to affect the eye (e.g. diabetes), visual acuity of less than $6 / 12$, and age over 80 years).

Thirty-nine eyes of 39 patients were included in the study. If both eyes of a patient were acceptable for inclusion in the study, one eye was selected at random. Statistical analysis was carried out wherever necessary using 'Easistat' software. Normal/abnormal criteria were based on our laboratory's standard control data obtained from 210 eyes of 210 normal volunteers. ${ }^{11}$

\section{RESULTS}

A summary of the data obtained on presentation from 39 eyes of 39 dysthyroid patients is presented in Table II. Five patients presented with dysthyroid optic nerve compression and a further 3 developed the condition during the review period.

No significant differences from normal were found for any of the patients in the non-compressive thyroid eye disease grades 1,2 and 3 (see Table III). Achromatic contrast sensitivity and chromatic discrimination sensitivity data obtained from individual eyes are presented graphically in Figs. 1a and 2a respectively.

Significant differences from normal were found for low frequency $(0.33$ and $0.66 \mathrm{cpd})$ achromatic contrast sensitivity in the grade 4 group (Table III). No significant difference from normal was found for any of the other parameters. Individual data obtained from patients in this group are presented graphically in Figs. $1 b$ and $2 b$. Achromatic contrast sensitivity for all spatial frequencies was found to be significantly different from normal in the grade 5 group (Table III). However, no significant difference in chromatic contrast sensitivity was found for either chromatic axis. Individual data obtained from patients in this group are presented graphically in Figs. 1c and 2c.

Significant reductions in all parameters were found in the grade 6 group (Table III). Individual data obtained from the 5 patients in this group are presented graphically in Figs. $1 \mathrm{~d}$ and $2 \mathrm{~d}$. The predominant decrease in colour sensitivity occurred in the tritan axis. One subject in the grade 6 group (Fig. 2d) was unusual in that she demonstrated a greater loss in the red/green axis on presentation with DON. Following orbital radiotherapy the red/ green deficit returned to normal, and at no time was Ishihara plate testing abnormal in this patient.

From Fig. $2 \mathrm{c}$ it is apparent that 3 individuals with grade 5 thyroid eye disease have abnormal chromatic discrimination sensitivity. Although these patients were clinically classified at presentation as having grade 5 thyroid eye disease, all 3 went on to develop clinically confirmed optic nerve compression that required treatment. We have found no correlation between the duration of thyroid eye disease and any of the test parameters.

\section{DISCUSSION}

We have found a consistent decrease in chromatic discrimination sensitivity in all cases of DON (i.e. NOSPECS grade 6). This decrease in sensitivity only occurred in those with DON and in patients with grade 5 disease who subsequently developed DON. It seems probable that those patients classified as having grade 5 disease were suffering from early, sub-clinical levels of optic nerve compression at the time of chromatic discrimination testing. The achromatic sensitivity was also decreased in all cases of DON; however, this test is less discriminatory since losses in low- and medium-frequency contrast sensitivity also occurred in those patients with grade 4 and grade 5 thyroid eye disease. This phenomenon has been described elsewhere, ${ }^{3,4,13,14}$ although the explanation for achromatic losses in the absence of optic nerve compression remains unclear.

Colour vision can be tested using the Ishihara plates, the American Optics (Hardy, Ritlor \& Rand) 


\section{a) Grades 1, 2 and 3}

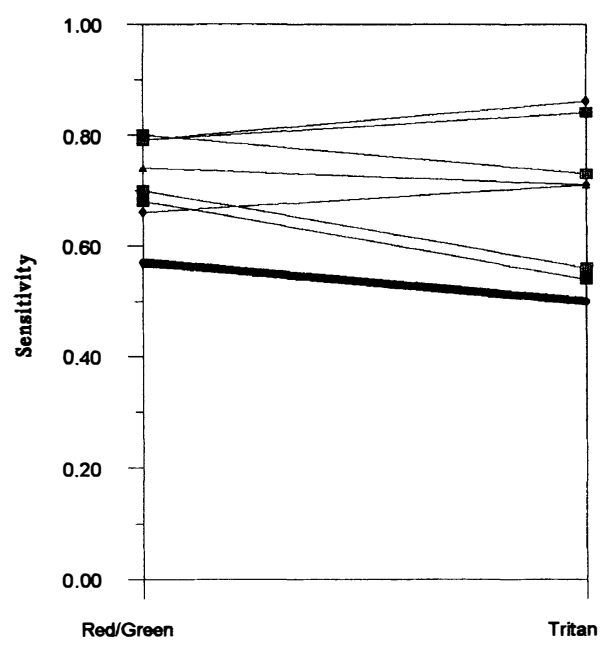

c) Grade 5

Corneal involvement

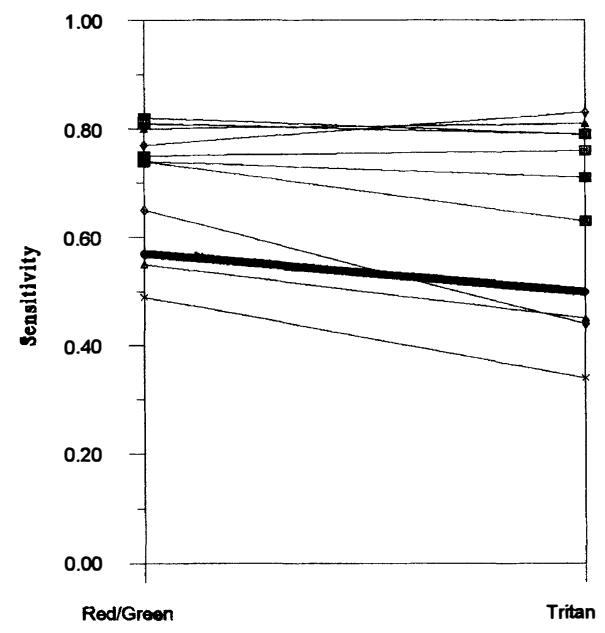

b) Grade 4

\section{Extraocular muscle involvement}

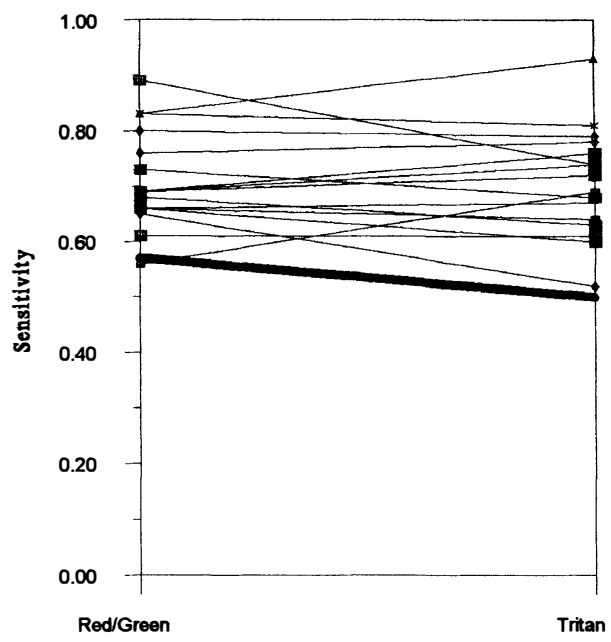

d) Grade 6

Dysthyroid optic neuropathy

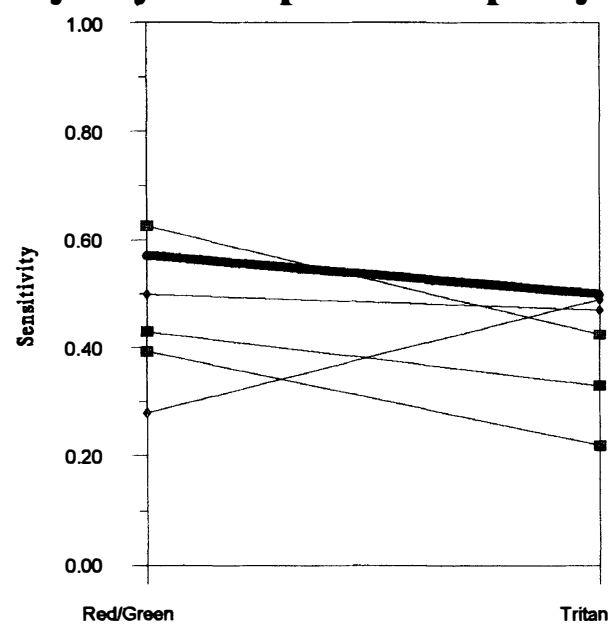

Fig. 1. Graphical representation of chromatic discrimination sensitivity data obtained from patients classified as having grade 1, 2 or 3 (a), grade 4 (b), grade 5 (c) or grade $6(d)$ dysthyroid eye disease as determined by the NOSPECS classification system. The bold lines represent age adjusted limits or normality.

plates and the Farnsworth-Munsell 100 hue test. However, these tests are designed to be used under very specific lighting conditions which are rarely available in clinical practice and, being printed tests, present problems with pigment degeneration. Furthermore, the Ishihara plates are designed primarily to detect congenital red/green colour vision deficiency and not the acquired, predominantly tritan axis deficits seen in DON.

It is reported that DON is bilateral in approximately $90 \%$ of cases. ${ }^{2}$ The RAPD is one of the few objective signs of asymmetric optic nerve compression but requires good examination technique, is difficult to grade, and is obviously of limited value in those with symmetrical disease processes such as DON. Chromatic discrimination sensitivity provides a definite, objective result for each eye and in our experience has often indicated bilateral optic nerve compression to a greater extent than that suspected on clinical examination alone.

The only other reference we have found regarding automated chromatic discrimination sensitivity testing in dysthyroid eye disease is a report by Potts $e t$ $a l .{ }^{6}$ describing a study comparing chromatic discrimination sensitivity, pattern ERGs, and cortical evoked potentials in the detection of DON. The report indicated that tritan chromatic discrimination losses as measured with Arden's automated system were found to be the most reliable indicator of the presence of DON.

The automated chromatic discrimination test described offers a quick and reliable confirmation 


\section{a) Grades 1, 2 and 3}

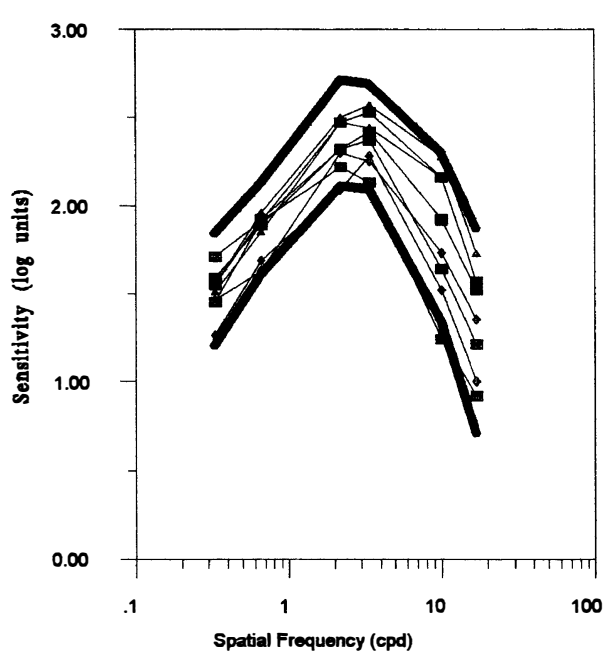

\section{c) Grade 5}

\section{Corneal involvement}

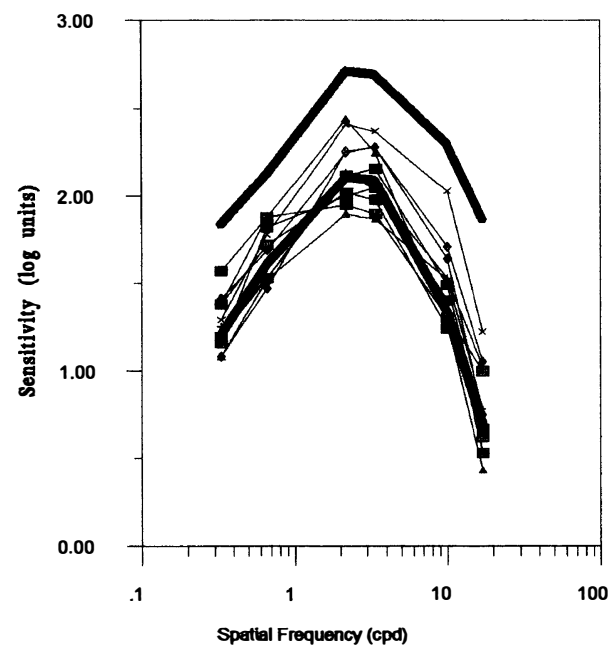

\section{b) Grade 4}

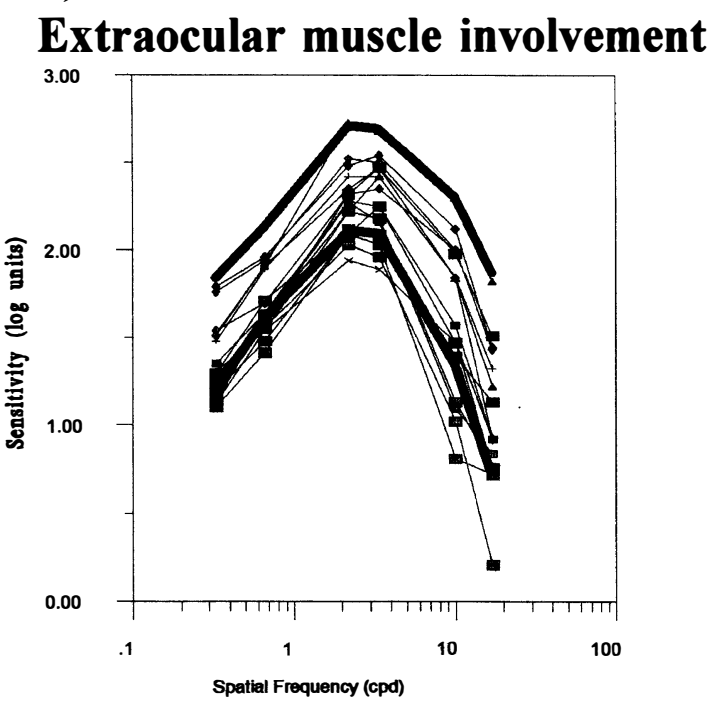

\section{d) Grade 6}

\section{Dysthyroid optic neuropathy}

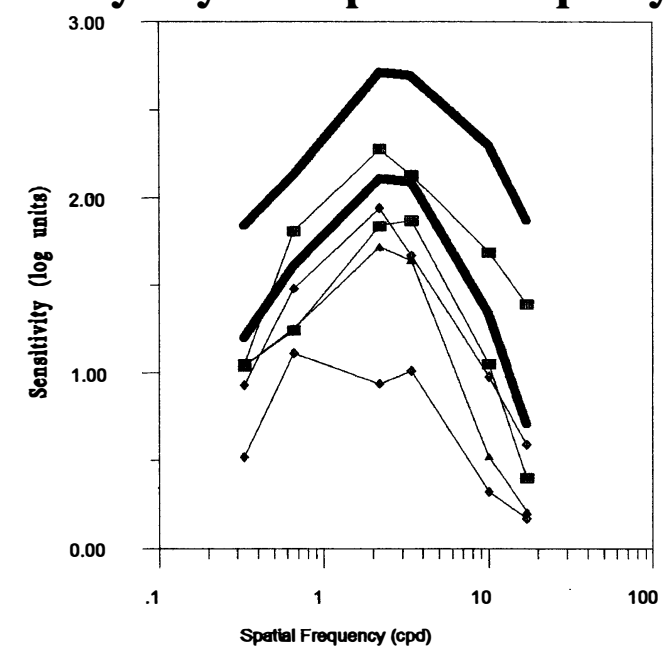

Fig. 2. Graphical representation of achromatic contrast sensitivity data obtained at presentation from patients with grades 1,2 or $3(a)$, grade $4(b)$, grade $5(c)$ and grade $6(d)$ dysthyroid eye disease as determined by the NOSPECS classification system. The bold lines represent the limits of normality.

of the early diagnosis of DON allowing referral for decompression to be made at an earlier stage of the disease process and with a greater level of confidence. We have also found the results useful in monitoring visual recovery following orbital decompression.

Further work is needed using larger patient numbers to evaluate whether the decompression of early DON, as detected by chromatic discrimination sensitivity testing, results in an improved visual prognosis and to elucidate the cause of the decreased achromatic sensitivity results seen in those dysthyroid patients who do not appear to have clinically recognised optic nerve compression.

Key words: Colour vision defects, Contrast sensitivity, Optic nerve diseases, Thyroid disease.

\section{REFERENCES}

1. Weetman AP. Thyroid-associated eye disease: pathophysiology. Lancet 1991;338:25-8.

2. Trobe JD, Glaser JS, Laflamme P. Dysthyroid optic neuropathy: clinical profile and rationale for management. Arch Ophthalmol 1978;96:1199-209.

3. Suttorp-Schulten MSA, Tijsen R, Mourits MPH, Apkarian P. Contrast sensitivity function in Graves' ophthalmopathy and dysthyroid optic neuropathy. $\mathrm{Br} \mathrm{J}$ Ophthalmol 1993;77:709-12.

4. Mourits MPH, Suttorp-Schulten MSA, Tijsen R, Apkarian P. Contrast sensitivity and the diagnosis of dysthyroid optic neuropathy. Doc Ophthalmol 1990; 74:329-35.

5. Weatherill J, Yap M. Recovery of contrast sensitivity following bilateral orbital decompression: a case report. Am J Optom Phys Optics 1985;62:715-9.

6. Potts MJ, Fells P, Falcao-Reis F, Bucetti S, Arden GB. Colour contrast sensitivity, pattern ERGs and cortical 
evoked potentials in dysthyroid optic neuropathy. Invest Ophthalmol Vis Sci 1990;31:189.

7. Werner SC. Classification of the eye changes of Graves' disease. J Clin Endocrinol Metab 1969;29:782.

8. de Alwis DV, Reffin JP, Tregear SJ, Casswell AG, Ripley LG. Should management of diabetic retinopathy be based upon measurements of visual function rather than observations of retinal morphology? Invest Ophthalmol Vis Sci 1993;34:719.

9. Reffin JP. The design and clinical application of tests of colour vision. DPhil thesis, University of Sussex, Falmer, Brighton,UK, 1991.

10. Tregear SJ, Knowles PJ, Ripley LG, Casswell AG. Colour vision deficits predict the development of severe diabetic retinopathy in diabetic subjects with background retinopathy. Invest Ophthalmol Vis Sci 1993;34:719.

11. Tregear SJ, Ripley LG, Knowles PJ, Gilday RT, de Alwis DV, Reffin JP. Automated Tritan Discrimination: a new clinical technique for the effective screening of severe diabetic retinopathy. Int $\mathbf{J}$ Psychophys 1994;16:191-8.

12. Cornsweet TN. The staircase method in psychophysics. Am J Psychol 1962;75:485-91.

13. Jindra LF, Zemon V. Contrast sensitivity testing: a more complete assessment of vision. J Cataract Refract Surg 1989;15:141-7.

14. Arden GB. Visual loss in patients with a normal visual acuity. Trans Ophthalmol Soc UK 1978;98:219-31. 